\title{
Comparison of the Modified Transtibial Technique, Anteromedial Portal Technique and Outside-in Technique in ACL Reconstruction
}

\author{
Oog Jin Sohn, MD, Dong Chul Lee, MD, Kang Hyun Park, MD, and Hyo Sae Ahn, MD \\ Department of Orthopedic Surgery, Yeungnam University Medical Center, Daegu, Korea
}

\begin{abstract}
Purpose: To compare clinical and radiographic results of anterior cruciate ligament (ACL) reconstruction using modified transtibial technique (mTT), anteromedial (AM) portal technique and outside-in (OI) technique.

Materials and Methods: From March 2007 to December 2012, ACL reconstruction was performed using the mTT, AM portal technique and OI technique in 20 patients each. Anteroposterior and rotational stability were assessed using the GNRB arthrometer and pivot-shift test. Femoral tunnel obliquity was measured on the anteroposterior and lateral radiographs.

Results: Tegner score, Lysholm score, International Knee Documentation Committee score, pivot-shift test were improved at final follow-up in all three groups. However, statistical difference was not shown. Side-to-side difference in GNRB arthrometer was average of $1.4 \mathrm{~mm}, 1.0 \mathrm{~mm}$ and 0.9 $\mathrm{mm}$ in $\mathrm{mTT}, \mathrm{AM}$ and OI group, showing the signicant difference between $\mathrm{mTT}$ and AM/OI groups $(\mathrm{p}<0.001)$. The mean femoral tunnel obliquity was $56.4^{\circ}$ in the mTT group, $39.4^{\circ}$ in the AM group and $33.6^{\circ}$ in the OI group, showing significant intergroup difference ( $\left.\mathrm{p}<0.001\right)$. It showed the significant difference among three groups $(\mathrm{p}<0.001)$.

Conclusions: Clinical results were improved in all three groups. Femoral tunnel obliquity in AM and OI were more horizontal on the coronal plane. They are thought to have more benefits in oblique positioning of the grafted ACL in rotational stability; however, there was no significant difference among three techniques in pivot-shift test to assess the rotational stability in this study.
\end{abstract}

Keywords: Knee, Anterior cruciate ligament, Reconstruction, Rotational stability

\section{Introduction}

The success rates of single-bundle reconstruction of the anterior cruciate ligament (ACL) have been reported to be approximately $83 \%-95 \%^{1,2}$. Single-bundle reconstruction is effective in restoring anteroposterior stability of the knee joint, but not in providing rotational stability due to the increased obliquity of the

Received December 17, 2013; Revised (1st) April 22, 2014;

(2nd) July 25, 2014; Accepted July 28, 2014

Correspondence to: Oog Jin Sohn, MD

Department of Orthopedic Surgery, Yeungnam University Medical

Center, 170 Hyeonchung-ro, Nam-gu, Daegu 705-717, Korea

Tel: +82-53-620-3640, Fax: +82-53-628-4020

E-mail: christiansio@hanmail.net

This is an Open Access article distributed under the terms of the Creative Commons Attribution Non-Commercial License (http://creativecommons.org/licenses/by-nc/3.0/) which permits unrestricted non-commercial use, distribution, and reproduction in any medium, provided the original work is properly cited. reconstructed $\mathrm{ACL}^{3-5)}$. Kato et al. ${ }^{6}$ reported that graft placement between the attachments of the anteromedial (AM) bundle and posterolateral bundle of the tibia and femur during bone tunneling was most effective for the recovery of stability and kinematics of the knee joint after single-bundle reconstruction. In addition, Yagi et al. ${ }^{7)}$ reported that close to normal biomechanics of the knee joint was obtained after anatomical reconstruction of the ACL.

Anatomical position and coronal obliquity of the ACL graft are important for restoration ofthe rotational stability and maintenence of the knee joint functions in the long term ${ }^{4,8-11)}$. In the previously commonly used transtibial technique, the position of the femoral tunnel was determined by the tibial tunnel, rendeing anatomical reconstruction difficult ${ }^{8,12-14)}$. Thus, the modified transtibial technique (mTT), the AM portal technique and the outside-in (OI) technique were proposed for anatomical bone tunnel placement ${ }^{15-17)}$. Since the latter two techniques result in more horizontal femoral tunnels than the mTT, they have been 
thought to bring greater improvement in the anteroposterior laxity and rotational stability. However, there have been few stuides focusing on the comparison of the clinical and radiographic results of ACL reconstruction performed using the three techniques. Therefore, in this study, under the hypothesis that the $\mathrm{AM}$ portal and $\mathrm{OI}$ techniques are more effective in restoring knee function and stability of the knee joint than the mTT because of the more horizontal coronal obliquity of the grafted ACL, we compared the clinical and radiographic results of ACL reconstrcution performed using these three techniques.

\section{Materials and Methods}

\section{Study Subjects}

Of 302 patients who underwent single-bundle ACL reconstruction from March 2007 to December 2012, 60 consecutive patients were enrolled in this prospective study. The ACL reconstruction was performed using the mTT technique in 20 patients ( 19 males and 1 female), AM portal technique in another 20 patients (19 males and 1 female) and OI technique in the other 20 patients (17 males and 3 females). The exclusion criteria were multiple ligament injuries, re-rupture of the anterior cruciate ligament, injury to the knee meniscus requiring total or subtotal meniscectomy, cartilage injury of Outerbridge grade IV or more and less than 1-year follow-up. An allogeneic tibialis anterior tendon was used as graft in all three groups. Their mean age was 29.8 years (range, 16 to 46 years), 26.9 years (range, 17 to 49 years) and 31.4 years (range, 15 to 51 years) in the mTT group, AM portal group and OI group, respectively. The mean follow-up period was 18.3 months (range, 13 to 27 months), 16.4 months (range, 12 to 25 months) and 12.1 months (range, 12 to 15 months) in the mTT group, AM portal group and OI group, respectively. At 3 months after injury, the mTT group had 12 patients with an acute injury and 8 patients with a chronic injury; the AM portal group had 12 patients with an acute injury and 8 patients with a chronic injury; and the OI group had 13 patients with an acute injury and 7 patients with a chronic injury. Sports injuries accounted for approximately $60 \%$ in all three groups. Body mass index was similar among three groups. There was no significant difference in patient demographics. Injury to the medial meniscus was most commonly noted, of which there was no significant difference in the treatment protocol (either partial resection or repair) (Table 1).

\section{Surgical Techniques}

1) Reconstruction using the mTT

In reconstruction using the $\mathrm{mTT}$, the tibial tunnel is drilled from the proximal and medial parts to lower the obliquity of the femoral tunnel compared to the conventional transtibial technique. In general, the starting point of the tibial tunnel is located midway between the posterior cortex of the proximal tibia and the medial margin of the tibial tuberosity. In order to avoid complete removal of the remnant ACL at the tibial attachment site and prevent impingement of the grafted ACL, minimal notchplasty was performed. Subsequently, an appropriate procedure for the accompanying meniscal or cartilage injury was carried out, if necessary. Following the tibial tunnel creation, a transtibial femoral tunnel guide was inserted through it and the rear angle of the guide was placed in the direction of 10:30 or 1:30 o'clock. Then, a tunnel with a depth of $30 \mathrm{~mm}$ and a diameter $1 \mathrm{~mm}$ less than that

Table 1. Patients Demographics

\begin{tabular}{lccc}
\hline \multicolumn{1}{c}{ Variable } & Modified transtibial & Anteromedial portal & Outside-in \\
\hline Age $(\mathrm{yr})$ & $29.5(16-46)$ & $26.9(17-49)$ & $31.4(15-51)$ \\
Sex (male:female) & $19: 1$ & $19: 1$ & $17: 3$ \\
Acute $(<3$ mo):chronic & $13: 7$ & $12: 8$ & $13: 7$ \\
Follow-up (mo) & $18.7(13-27)$ & 65 & $12.1(12-15)$ \\
Sports injury (\%) & 60 & 26.1 & 60 \\
Body mass index $\left(\mathrm{kg} / \mathrm{m}^{2}\right)$ & 25.7 & & 25.2 \\
Combined injury & & 9 & 3 \\
MM & 9 & 3 & 3 \\
LM & 5 & 3 & 3 \\
MM+LM & 2 & & 3 \\
\hline
\end{tabular}

Values are presented as mean (range). p-value $>0.05$.

MM: medial meniscus, LM: lateral meniscus. 
of the graft was drilled to achieve approximately $1-2 \mathrm{~mm}$ of the femoral posterior wall. The tunnel with the final diameter was completed by using a dilator with an interval of $0.5 \mathrm{~mm}$, and the graft was fixed using the Endobutton CL (Smith \& Nephew Inc., Andover, MA, USA). The tibial tunnel was fixed again by using a post-tie after it was fixed with a bioabsorbable interference screw.

\section{2) Reconstruction using the AM portal technique}

In order to form an AM portal in the appropriate location, a spinal needle was inserted just above the anterior horn of the medial meniscus of the knee under the arthroscope with the knee joint in $90^{\circ}$ flexion. After examining whether it had passed through the anterior cartilage margin of the medial femoral condyle and oriented toward the anatomical origin of the medial wall of the lateral femoral condyle, the insertion site was formed. Then, the femoral tunnel was created with the knee joint in $110^{\circ}-130^{\circ}$ flexion by using the Transportal Guide (Arthrex Inc., Naples, FL, USA). The tibial tunnel was created in the same way as in the transtibial technique. The femoral tunnel was fixed by using the Endobutton CL and the tibial tunnel was fixed by using a bioabsorbable interference screw and a post-tie.

\section{3) The reconstruction using the OI technique}

A central midpatellar portal was made at the patellar tendon to introduce the RetroConstruction Drill Guide (Arthrex Inc.) after marked the point of entry with an Arthrocare device (Arthrocare Corp., Austin, TX, USA) and a Steadman awl on the center of the AM and PL footprint. Then, a skin incision was performed on the lateral femoral condyle and the femoral tunnel was formed by retrograde reaming using FlipCutter (Arthrex Inc.). In addition, after the tibial tunnel was formed in the same way as in the the transtibial technique and the AM portal technique, the graft was passed through the tunnel and then the femoral tunnel was fixed with TightRope (Arthrex Inc.) The tibial tunnel was fixed by using a bioabsorbable interference screw and a post-tie.

\section{Postoperative Rehabilitation}

The postoperative rehabilitation protocol was the same in all three groups. Joint flexion and extension were allowed starting from the day after the surgery. In patients who did not undergo a meniscal repair procedure, partial weight bearing was performed for 2 weeks while wearing the ACL brace. After 2 weeks, full weight bearing was allowed. In patients who had meniscal repair, partial weight bearing was performed for 6 weeks and the brace was worn for 6 weeks. Jogging was allowed from 3 months after surgery. Sports activity was allowed from $6-9$ months after sur-
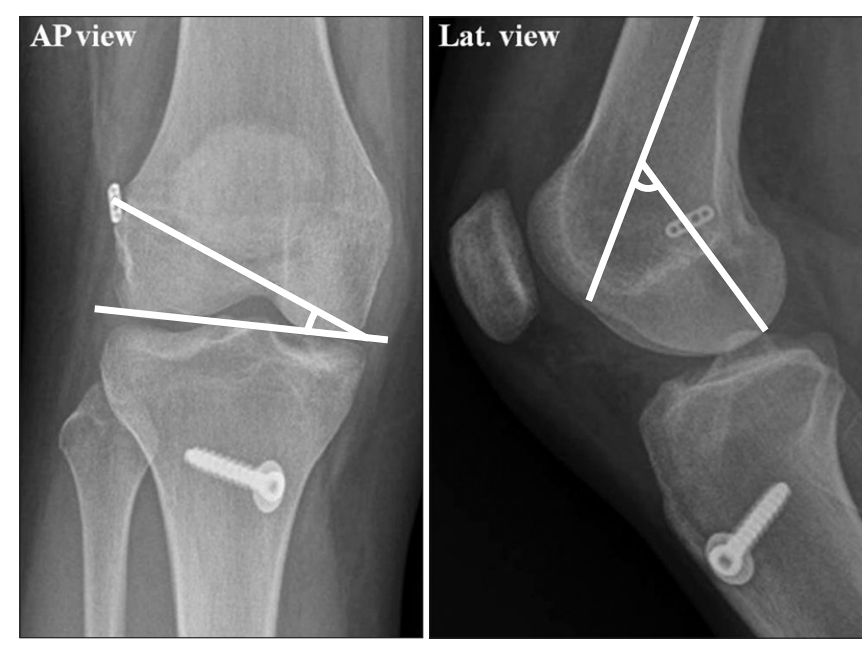

Fig. 1. Femoral tunnel angles were measured on the postoperative anteroposterior (AP) and lateral (Lat) radiographs. On the AP radiograph, the angle between the femoral tunnel and a line parallel to the articular surface was measured. On the Lat radiograph, the angle between the femoral tunnel and an extension line of the posterior cortex of the femoral shaft was measured.

gery depending on the state of recovery.

\section{Clinical and Radiographic Assessment and Statistical Analysis}

Clinical results were assessed using Tegner activity score, Lysholm knee joint score, and International Knee Documentation Committee (IKDC) subjective scores preoperatively and at final follow-up. Anteroposterior and rotational stability was measured using the GNRB arthrometer (GeNouRoB, Laval, France) and pivot-shift test. Femoral tunnel obliquity was also measured. On the anteroposterior radiograph, the angle between the femoral tunnel and a line parallel to the articular surface was measured. On the lateral radiograph, the angle between the femoral tunnel and the extension line of the posterior cortex of the femur shaft was measured (Fig. 1). Anterior displacement of the tibia was examined using the anterior drawer radiograph. Statistical analysis of research data was performed by using SPSS ver. 19.0 (IBM Corp., Armonk, NY, USA) statistical program. The results of ACL reconstruction in three groups were verified with posthoc test after analysis of variance test. A p-value of $<0.05$ was considered statistically significant.

\section{Results}

According to the clinical results, the Tegner activity scores in the mTT group were improved from an average of 2.5 points preoperatively to an average of 6.0 points at final follow-up. The 
Tegner activity scores were also improved from an average of 2.8 and 2.7 points preoperatively to an average of 6.3 and 6.5 points at final follow-up in the AM portal group and OI group, respectively. However, there was no statistically significant intergroup difference ( $p=0.124)$ (Fig. 2). The Lysholm scores in the mTT group were improved from an average of $52.2 \pm 12.2$ points preop-

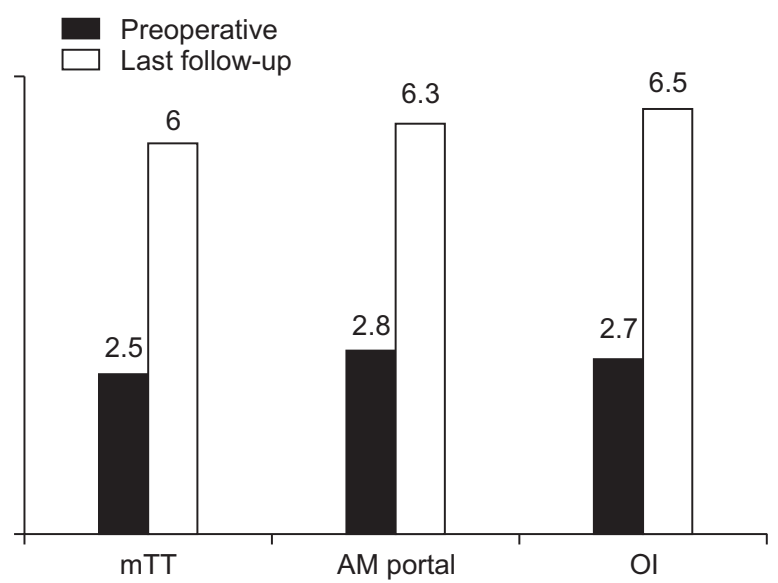

Fig. 2. The bar graph shows that there is no statistically significant difference between the three techniques with respect to changes in the Tegner activity level between the preoperative and last follow-up measurements. mTT: modified transtibial technique, AM: anteromedial, OI: outside-in. eratively to an average of $86.5 \pm 3.3$ points at final follow-up. The Lysholm scores were also improved from an average of $54.3 \pm 12.3$ points and $53.7 \pm 13.3$ points preoperatively to an average of $88.6 \pm 2.6$ points and $89.1 \pm 3.1$ points at final follow-up in the AM portal group and OI group, respectively. However, there was no significant intergroup difference $(\mathrm{p}=0.171)$ (Table 2$)$. The IKDC

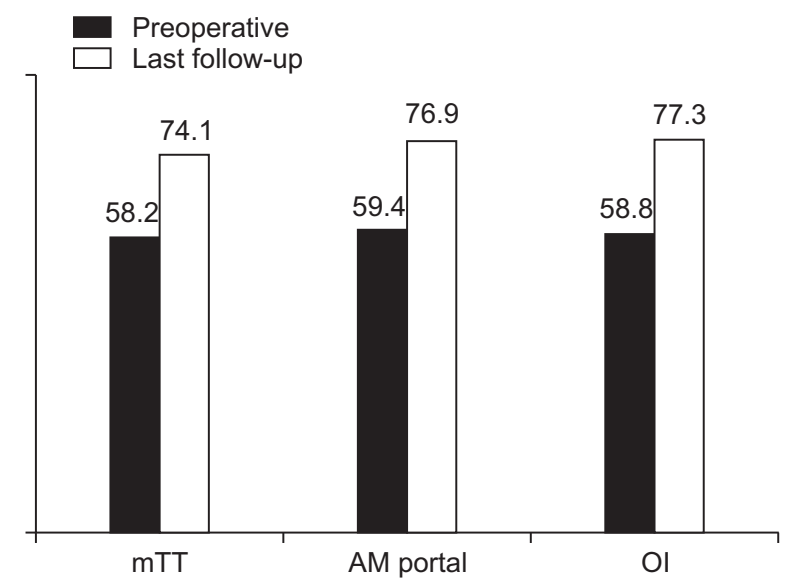

Fig. 3. The bar graph shows that there is no statistically significant difference between the three techniques with respect to changes in the International Knee Documentation Committee subjective score between the preoperative and the last follow-up measurements. mTT: modified transtibial technique, AM: anteromedial, OI: outside-in.

Table 2. Changes in the Lysholm Score

\begin{tabular}{|c|c|c|c|c|c|c|}
\hline \multirow{2}{*}{ Lysholm score } & \multicolumn{2}{|c|}{ Modified transtibial } & \multicolumn{2}{|c|}{ Anteromedial portal } & \multicolumn{2}{|c|}{ Outside-in } \\
\hline & Preop & Last F/U & Preop & Last F/U & Preop & Last F/U \\
\hline Excellent (95-100) & 0 & $3(15)$ & 0 & $4(20)$ & 0 & $5(25)$ \\
\hline Good (84-94) & 0 & $15(75)$ & 0 & $16(80)$ & 0 & $15(75)$ \\
\hline Fair (65-83) & $6(30)$ & $2(10)$ & $5(25)$ & 0 & $6(30)$ & 0 \\
\hline Poor (0-64) & $14(70)$ & 0 & $15(75)$ & 0 & $14(70)$ & 0 \\
\hline Mean score & 53.2 & 87.7 & 54.3 & 88.6 & 53.7 & 89.1 \\
\hline
\end{tabular}

Values are presented as number (\%). p-value $>0.05$.

Preop: preoperative, F/U: follow-up.

Table 3. Changes in the Pivot-Shift Test Results

\begin{tabular}{|c|c|c|c|c|c|c|}
\hline \multirow{2}{*}{ Grade } & \multicolumn{2}{|c|}{ Modified transtibial } & \multicolumn{2}{|c|}{ Anteromedial portal } & \multicolumn{2}{|c|}{ Outside-in } \\
\hline & Preop & Last F/U & Preop & Last F/U & Preop & Last $\mathrm{F} / \mathrm{U}$ \\
\hline 0 (negative) & $4(20)$ & $15(75)$ & $4(20)$ & $18(90)$ & $3(15)$ & $19(95)$ \\
\hline 1+ (glide) & $9(45)$ & $3(15)$ & $9(45)$ & $2(10)$ & $10(50)$ & $1(5)$ \\
\hline $2+($ clunk $)$ & $6(30)$ & $2(10)$ & $5(25)$ & 0 & $6(30)$ & 0 \\
\hline $3+$ (gross) & $1(5)$ & 0 & $2(10)$ & 0 & $1(5)$ & 0 \\
\hline
\end{tabular}

Values are presented as number (\%). p-value $>0.05$.

Preop: preoperative, F/U: follow-up. 
subjective scores were improved at final follow-up by an average of 15.4 points, 17.5 points and 18.5 points in the mTT group, AM portal group and OI group, respectively, compared to the preoperative values. However, no significant difference was noted $(\mathrm{p}=0.211)$ (Fig. 3). On the pivot-shift test, the mTT group exhibited improvement:18 cases (90\%) had less than $1+$ grade and 2 cases had $2+$ grade at final follow-up. The AM portal group and OI group exhibited improvements with all cases having less than $1+$ grade. However, no statistical significance intergroup was found $(\mathrm{p}=0.083)$ (Table 3). Regarding the evaluation of the anteroposterior stability of the knee joint in the GNRB arthrometer test, the side-to-side difference at final follow-up was an average of 1.4, 1.0, and $0.9 \mathrm{~mm}$ in the mTT group, AM portal group and OI group, respectively. There was no significant difference between the AM portal group and the OI group $(\mathrm{p}=0.225)$. However, statistically significant difference was noted between the mTT group and the

Table 4. GNRB Arthrometer Test Results

\begin{tabular}{lccc}
\hline GNRB arthrometer & $\begin{array}{c}\text { Modified } \\
\text { transtibial }\end{array}$ & $\begin{array}{c}\text { Anteromedial } \\
\text { portal }\end{array}$ & Outside-in \\
\hline Healthy & $4.5 \pm 1.9$ & $4.6 \pm 1.8$ & $4.5 \pm 1.5$ \\
Operated & $5.9 \pm 2.2$ & $5.6 \pm 1.9$ & $5.4 \pm 1.2$ \\
Mean difference & 1.4 & 1.0 & 0.9 \\
\hline
\end{tabular}

Values are presented as mean \pm standard deviation or number. p-value $>0.05$.
AM portal group/OI group ( $\mathrm{p}=0.000)$ (Table 4).

The coronal obliquity of the grafted ACL was $56.4^{\circ} \pm 9.6^{\circ}$, $39.4^{\circ} \pm 7.2^{\circ}$ and $33.6^{\circ} \pm 6.6^{\circ}$ in the mTT group, AM portal group and OI group, respectively. The obliquity was the most horizontal in the OI group and there were significant differences among three groups ( $\mathrm{p}=0.000$ ) (Fig. 4). The obliquity of the femoral tunnel on the sagittal plane was $49.4^{\circ} \pm 6.3^{\circ}, 50.9^{\circ} \pm 7.2^{\circ}$ and $52.1^{\circ} \pm 6.9^{\circ}$ in the mTT group, AM portal group and OI group, respectively, showing no significant difference among three groups $(\mathrm{p}=0.300)$ (Table 5). According to the anterior drawer radiographs, the mean difference in the anterior displacement of the tibia between the operated and the intact knees was 1.1, 1.0, and $0.9 \mathrm{~mm}$ in the mTT group, AM portal group and OI group, respectively, and no significant intergroup difference was found $(\mathrm{p}=0.255)$.

\section{Discussion}

The ACL consists of the AM bundle and the posterolateral bundle. Since the AM bundle is mainly responsible for anteroposterior stability and the posterolateral bundle is for rotational stability, the interaction of the two bundles contributes to the stability and kinematics of the knee joint ${ }^{9,18)}$. Regarding ACL reconstruction, there is still disagreement on whether double-bundle reconstruction is superior to single-bundle reconstruction for restoring stability or kinematics of the knee. Recent clinical studies have reported that double-bundle reconstruction provides
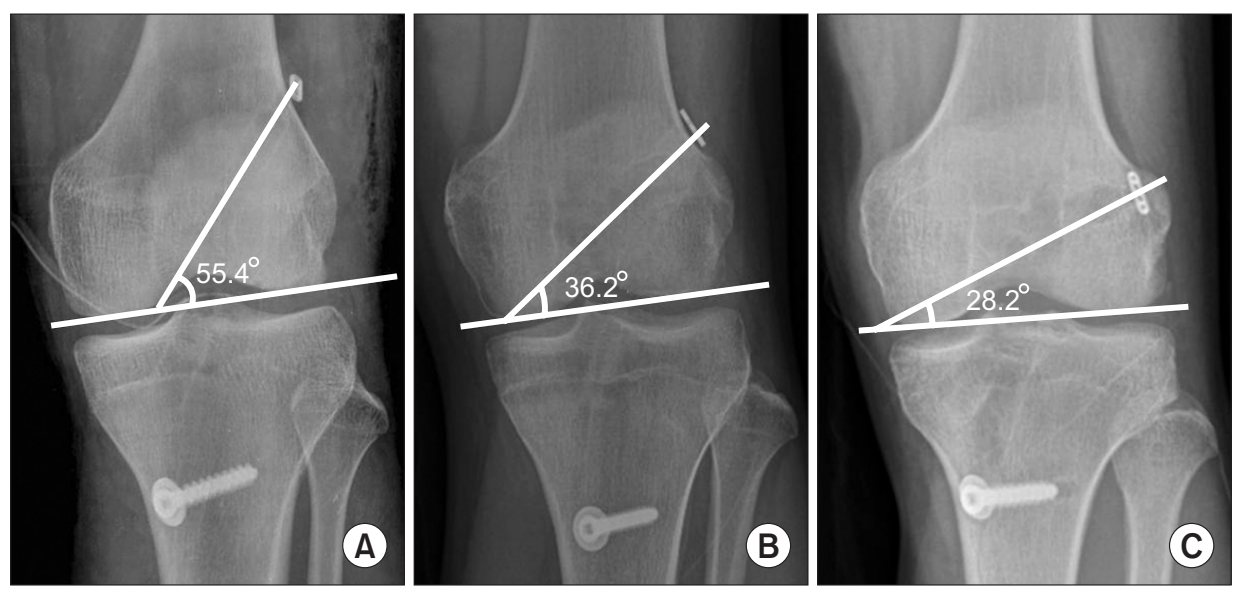

Fig. 4. The last follow-up anteroposterior radiographs show the femoral tunnel angles created using mTT (A), AM portal technique (B) and OI technique (C). The mean angle was significantly different in three groups $(p=0.000)$. Compared to the mTT, the AM portal technique and the OI technique would be more advantageous in obtaining oblique and anatomical femoral tunnels. mTT: modified transtibial technique, AM: anteromedial, OI: outside-in.

Table 5. Femoral Tunnel Angles

\begin{tabular}{|c|c|c|c|c|}
\hline Parameter $\left({ }^{\circ}\right)$ & Modified transtibial & Anteromedial portal & Outside-in & p-value \\
\hline Anteroposterior view & $56.4 \pm 9.6$ & $39.4 \pm 7.2$ & $33.6 \pm 6.6$ & 0.000 \\
\hline Lateral view & $49.4 \pm 6.3$ & $50.9 \pm 7.3$ & $52.1 \pm 6.9$ & $>0.05$ \\
\hline
\end{tabular}

Values are presented as mean \pm standard deviation. 
improved postoperative stability compared to single-bundle reconstruction ${ }^{19-21)}$. However, Kato et al. ${ }^{6}$ reported that anatomic mid-position single-bundle reconstruction was the most effective for the recovery of the stability and kinematics of the knee joint. Kim et al. ${ }^{22)}$ reported that rotational stability was restored close to the normal level after anatomic single-bundle reconstruction using two AM portals. Markolf et al. ${ }^{23)}$ reported that single-bundle reconstruction using the AM bundle was sufficient to normalize the biomechanics of the knee joint and raised a question whether double-bundle reconstruction, which is technically difficult and requires a longer operating time, is necessary.

For isometric femoral tunnel placement, which causes minimal changes in the length of graft, the direction of 11 oclock for the right knee or 1 oclock for the left knee has been considered as the standard for formation of the femoral tunnel ${ }^{9,24)}$. The location of the femoral tunnel is determined by the tibial tunnel in the transtibial technique. Since it allows for isometric femoral tunnel placement, the technique has been most commonly utilized in ACL reconstruction. However, Arnold et al. ${ }^{12)}$ reported that it may not be effective in lowering the femoral tunnel obliquity which is dependent on that of a tibial tunnel. It has been reported that accurate femoral tunnel placement is one of the most important procedures for obtaining good clinical results in ACL reconstruction and the obliquity of the femoral tunnel is important for the recovery of rotational stability ${ }^{9}$. Jepsen et al. ${ }^{4)}$ reported that the position of the femoral tunnel in the direction of 10 oclock resulted in greater clinical improvement and higher patient satisfaction than the 11 oclock position. Scopp et al. ${ }^{10)}$ reported that rotational stability was restored close to the normal level when the femoral tunnel was located lower on the coronal plane. Therefore, in an attempt to create a more horizontal femoral tunnel, we used the mTT, AM portal, and OI techniques in ACL reconstruction.

Clinically, Tegner activity score, Lysholm score and IKDC score did not show statistical significant difference between the techniques, but the AM portal and OI techniques showed slightly better results than the mTT technique. On the anteroposterior stability assessment, Kim et al. ${ }^{25)}$ and Panni et al. ${ }^{26)}$ reported that there was no significant difference between two different techniques for ACL reconstruction based on the side-to-side difference values obtained using the KT-2000 arthrometer. In the current study, the anteroposterior stability was assessed by using the GNRB arthrometer and the decrease in anterior displacement was significantly greater in the AM portal group and the OI group than in the mTT group.

Regarding the fermoral tunnel obliquity on the coronal plane,
Takeda et al. ${ }^{27)}$ showed that the obliquity was greater in the transtibial technique group than in the $\mathrm{AM}$ portal group $\left(59.0^{\circ} \pm 8.0^{\circ}\right.$ vs. $38.0^{\circ} \pm 6.0^{\circ}$ ). Panni et al..$^{26)}$ reported that the value was greater in the transtibial group than in the OI group $\left(65.4^{\circ} \pm 7.6^{\circ}\right.$ vs. $\left.42.2^{\circ} \pm 7.9^{\circ}\right)$. In our study, the mTT group, the AM portal and the OI group had a mean femoral tunnel obliquity of $56.4^{\circ} \pm 9.6^{\circ}$, $39.4^{\circ} \pm 7.2^{\circ}$ and $33.6^{\circ} \pm 6.6^{\circ}$, respectively; thus, the femoral tunnel was most horizontal in the OI group. However, no significant difference was observed between these techniques in the pivot-shift test for rotational stability. In the mTT group, sufficiently long tibial tunnels were established without careless verticalization of the tunnel. In the AM portal group, the tibial tunnel was formed independently. Thus, no significant difference was noted in the evaluation of rotational stability. We believe larger cases and longer follow-up are needed to confirm the results. In order to lower the obliquity of the femoral tunnel in the transtibial technique, Rue et al. ${ }^{28)}$ moved the starting point of the tibial tunnel more proximally and medially compared to the conventional method and performed femoral tunnel placement in the direction of 10 or 2 oclock, if possible. However, this modification may cause damage to the the medial collateral ligament and pes anserinus and problems during fixation and healing of graft due to the short tibial tunnel length.

It has been reported that the AM portal technique is advantageous for anteroposterior stability since the femoral tunnel can be created close to the anatomical position and more horizontally on the coronal plane. It has been suggested as a useful technique to place the graft in the anatomical position ${ }^{15}$. However, without hyperflexion of the knee, it may result in damage to the posterior wall of the lateral femoral condyle upon forming the femoral tunnel and creation of a short femoral tunnel $(<30 \mathrm{~mm})$, which is disadvantageous for fixation and healing of the graft. Therefore, the knee should be hyperflexed more than $110^{\circ}$ during femoral tunnel drilling to avoid complications, which could also limit the field of view during surgery ${ }^{29)}$.

The OI technique has some advantages including more uniform femoral tunnel placement, good visibility during surgery and sufficient femoral tunnel length compared to the AM portal technique ${ }^{17)}$.

One of the limitations of this study is the relatively short followup period that ranges from 12.1 to 18.7 months. We think that more than 5-year follow-up is necessary for more accurate assessment of the stability of the knee joint. In addition, besides the pivot-shift test that was used to assess the rotational stability, quantitative tests should also be performed to improve the accuracy of the results. Finally, except for the analysis on the angles 
of the femoral tunnels on simple radiographs, assessments on the obliquity of grafts and position \& length of bone tunnels, and angles were not adressed in this study. In our opinion, more structural assessments using three dimensional computed tomography or magnetic resonance imaging are necessay.

\section{Conclusions}

There was no statistically significant difference between the mTT, AM portal, and OI techniques based on the clinical results of single-bundle ACL reconstruction, but the latter two techniques demonstrated slightly better clinical results. In addition, since the AM portal technique resulted in more horizontal obliquity of the femoral tunnel than the OI technique, it could be more helpful for oblique positioning of the ACL graft for rotational stability. However, no statistically significant difference was noted between the three techniques in the pivot-shift test for rotational stability.

\section{Conflict of Interest}

No potential conflict of interest relevant to this article was reported.

\section{References}

1. Feller JA, Webster KE. A randomized comparison of patellar tendon and hamstring tendon anterior cruciate ligament reconstruction. Am J Sports Med. 2003;31:564-73.

2. Seon JK, Park SJ, Lee KB, Yoon TR, Seo HY, Song EK. Stability comparison of anterior cruciate ligament between double- and single-bundle reconstructions. Int Orthop. 2009;33: 425-9.

3. Aglietti P, Giron F, Cuomo P, Losco M, Mondanelli N. Single-and double-incision double-bundle ACL reconstruction. Clin Orthop Relat Res. 2007;454:108-13.

4. Jepsen CF, Lundberg-Jensen AK, Faunoe P. Does the position of the femoral tunnel affect the laxity or clinical outcome of the anterior cruciate ligament-reconstructed knee? A clinical, prospective, randomized, double-blind study. Arthroscopy. 2007;23:1326-33.

5. Kanamori A, Zeminski J, Rudy TW, Li G, Fu FH, Woo SL. The effect of axial tibial torque on the function of the anterior cruciate ligament: a biomechanical study of a simulated pivot shift test. Arthroscopy. 2002;18:394-8.

6. Kato Y, Ingham SJ, Linde-Rosen M, Smolinski P, Horaguchi
T, Fu FH. Biomechanics of the porcine triple bundle anterior cruciate ligament. Knee Surg Sports Traumatol Arthrosc. 2010;18:20-5.

7. Yagi M, Wong EK, Kanamori A, Debski RE, Fu FH, Woo SL. Biomechanical analysis of an anatomic anterior cruciate ligament reconstruction. Am J Sports Med. 2002;30:660-6.

8. Heming JF, Rand J, Steiner ME. Anatomical limitations of transtibial drilling in anterior cruciate ligament reconstruction. Am J Sports Med. 2007;35:1708-15.

9. Loh JC, Fukuda Y, Tsuda E, Steadman RJ, Fu FH, Woo SL. Knee stability and graft function following anterior cruciate ligament reconstruction: Comparison between 11 oclock and 10 o'clock femoral tunnel placement: 2002 Richard O’Connor Award paper. Arthroscopy. 2003;19:297-304.

10. Scopp JM, Jasper LE, Belkoff SM, Moorman CT 3rd. The effect of oblique femoral tunnel placement on rotational constraint of the knee reconstructed using patellar tendon autografts. Arthroscopy. 2004;20:294-9.

11. Alentorn-Geli E, Samitier G, Alvarez P, Steinbacher G, Cugat R. Anteromedial portal versus transtibial drilling techniques in ACL reconstruction: a blinded cross-sectional study at two- to five-year follow-up. Int Orthop. 2010;34:747-54.

12. Arnold MP, Kooloos J, van Kampen A. Single-incision technique misses the anatomical femoral anterior cruciate ligament insertion: a cadaver study. Knee Surg Sports Traumatol Arthrosc. 2001;9:194-9.

13. Kaseta MK, DeFrate LE, Charnock BL, Sullivan RT, Garrett WE Jr. Reconstruction technique affects femoral tunnel placement in ACL reconstruction. Clin Orthop Relat Res. 2008;466:1467-74.

14. Kopf S, Forsythe B, Wong AK, Tashman S, Anderst W, Irrgang JJ, Fu FH. Nonanatomic tunnel position in traditional transtibial single-bundle anterior cruciate ligament reconstruction evaluated by three-dimensional computed tomography. J Bone Joint Surg Am. 2010;92:1427-31.

15. Harner CD, Poehling GG. Double bundle or double trouble? Arthroscopy. 2004;20:1013-4.

16. Lubowitz JH. Anteromedial portal technique for the anterior cruciate ligament femoral socket: pitfalls and solutions. Arthroscopy. 2009;25:95-101.

17. Lubowitz JH, Akhavan S, Waterman BR, Aalami-Harandi A, Konicek J. Technique for creating the anterior cruciate ligament femoral socket: optimizing femoral footprint anatomic restoration using outside-in drilling. Arthroscopy. 2013;29: 522-8.

18. Mommersteeg TJ, Huiskes R, Blankevoort L, Kooloos JG, 
Kauer JM. An inverse dynamics modeling approach to determine the restraining function of human knee ligament bundles. J Biomech. 1997;30:139-46.

19. Kondo E, Yasuda K, Azuma H, Tanabe Y, Yagi T. Prospective clinical comparisons of anatomic double-bundle versus single-bundle anterior cruciate ligament reconstruction procedures in 328 consecutive patients. Am J Sports Med. 2008; 36:1675-87.

20. Jarvela T. Double-bundle versus single-bundle anterior cruciate ligament reconstruction: a prospective, randomize clinical study. Knee Surg Sports Traumatol Arthrosc. 2007;15: 500-7.

21. Yagi M, Kuroda R, Nagamune K, Yoshiya S, Kurosaka M. Double-bundle ACL reconstruction can improve rotational stability. Clin Orthop Relat Res. 2007;454:100-7.

22. Kim JG, Wang JH, Lim HC, Ahn JH. Femoral graft bending angle and femoral tunnel geometry of transportal and outside-in techniques in anterior cruciate ligament reconstruction: an in vivo 3-dimensional computed tomography analysis. Arthroscopy. 2012;28:1682-94.

23. Markolf KL, Park S, Jackson SR, McAllister DR. Simulated pivot-shift testing with single and double-bundle anterior cruciate ligament reconstructions. J Bone Joint Surg Am. 2008;90:1681-9.

24. Lee MC, Seong SC, Lee S, Chang CB, Park YK, Jo H, Kim $\mathrm{CH}$. Vertical femoral tunnel placement results in rotational knee laxity after anterior cruciate ligament reconstruction.
Arthroscopy. 2007;23:771-8.

25. Kim MK, Lee BC, Park JH. Anatomic single bundle anterior cruciate ligament reconstruction by the two anteromedial portal method: the comparison of transportal and transtibial techniques. Knee Surg Relat Res. 2011;23:213-9.

26. Panni AS, Milano G, Tartarone M, Demontis A, Fabbriciani C. Clinical and radiographic results of ACL reconstruction: a 5- to 7-year follow-up study of outside-in versus insideout reconstruction techniques. Knee Surg Sports Traumatol Arthrosc. 2001;9:77-85

27. Takeda Y, Iwame T, Takasago T, Kondo K, Goto T, Fujii K, Naruse A. Comparison of tunnel orientation between transtibial and anteromedial portal techniques for anatomic double-bundle anterior cruciate ligament reconstruction using 3-dimensional computed tomography. Arthroscopy. 2013;29:195-204.

28. Rue JP, Ghodadra N, Bach BR Jr. Femoral tunnel placement in single-bundle anterior cruciate ligament reconstruction: a cadaveric study relating transtibial lateralized femoral tunnel position to the anteromedial and posterolateral bundle femoral origins of the anterior cruciate ligament. Am J Sports Med. 2008;36:73-9.

29. Zantop T, Herbort M, Raschke MJ, Fu FH, Petersen W. The role of the anteromedial and posterolateral bundles of the anterior cruciate ligament in anterior tibial translation and internal rotation. Am J Sports Med. 2007;35:223-7. 